\title{
Article
}

\section{Reconstitution of Cytokinin Signaling in Rice Protoplasts}

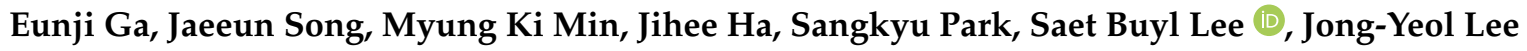 \\ and Beom-Gi Kim *
}

Citation: Ga, E.; Song, J.; Min, M.K.; Ha, J.; Park, S.; Lee, S.B.; Lee, J.-Y.;

Kim, B.-G. Reconstitution of

Cytokinin Signaling in Rice

Protoplasts. Int. J. Mol. Sci. 2021, 22,

3647. https://doi.org/10.3390/

ijms22073647

Academic Editor: Georgy

A. Romanov

Received: 26 February 2021

Accepted: 29 March 2021

Published: 31 March 2021

Publisher's Note: MDPI stays neutral with regard to jurisdictional claims in published maps and institutional affiliations.

Copyright: (C) 2021 by the authors. Licensee MDPI, Basel, Switzerland. This article is an open access article distributed under the terms and conditions of the Creative Commons Attribution (CC BY) license (https:/ / creativecommons.org/licenses/by/ $4.0 /)$.
Metabolic Engineering Division, National Institute of Agricultural Sciences, RDA, Jeonju 54874, Korea; vapofi@naver.com (E.G.); icanje@korea.kr (J.S.); mkmin66@korea.kr (M.K.M.); hjh10942@korea.kr (J.H.); psk2779@korea.kr (S.P.); buylee@korea.kr (S.B.L.); jy0820@korea.kr (J.-Y.L.)

* Correspondence: bgkimpeace@gmail.com

\begin{abstract}
The major components of the cytokinin (CK) signaling pathway have been identified from the receptors to their downstream transcription factors. However, since signaling proteins are encoded by multigene families, characterizing and quantifying the contribution of each component or their combinations to the signaling cascade have been challenging. Here, we describe a transient gene expression system in rice (Oryza sativa) protoplasts suitable to reconstitute CK signaling branches using the CK reporter construct TCSn:fLUC, consisting of a synthetic CK-responsive promoter and the firefly luciferase gene, as a sensitive readout of signaling output. We used this system to systematically test the contributions of CK signaling components, either alone or in various combinations, with or without CK treatment. The type-B response regulators (RRs) OsRR16, OsRR17, OsRR18, and OsRR19 all activated TCSn:fLUC strongly, with OsRR18 and OsRR19 showing the strongest induction by CK. Cotransfecting the reporter with OsHP01, OsHP02, OsHP05, or OsHK03 alone resulted in much weaker effects relative to those of the type-B OsRRs. When we tested combinations of OsHK03, OsHPs, and OsRRs, each combination exhibited distinct CK signaling activities. This system thus allows the rapid and high-throughput exploration of CK signaling in rice.
\end{abstract}

Keywords: rice; protoplast; cytokinin; signaling; reconstitution

\section{Introduction}

Plants interact with their environments via the perception of physical and chemical signals, such as light, temperature, and nutrients. Among the multiple mechanisms employed by plants to respond to their surroundings, phytohormones are endogenous chemical signals that take on a prominent role to modulate and coordinate plant growth and development by integrating the external environment and intracellular programs [1,2]. Each phytohormone is recognized by a specific and distinct receptor that then transduces the signal to its downstream cognate signaling components [2-4].

Phytohormone signaling cascades are complex due to redundancy among signaling components, which are typically encoded by multigene families, as well as multilayered effects among phytohormones [4]. Thus, a quantitative dissection of the individual contribution of each signaling component has proven difficult because loss-of-function alleles in single genes within a gene family very often cause no obvious alterations in phenotype, thus necessitating the generation of higher-order loss-of-function mutant series to reveal their underlying functions [5,6]. Gain-of-function approaches may be used as an alternative method to characterize the effects associated with individual genes on a given signaling pathway via stable or transient gene overexpression, although the resulting higher levels of the protein under study may not reflect biological functions accurately $[7,8]$.

Transient gene expression systems are powerful methods, as they allow rapid and large-scale screening of signaling components, despite the potential limitations mentioned above. Accordingly, heterologous systems, such as yeast (Saccharomyces cerevisiae), and homologous systems for transient gene expression systems, such as protoplasts and Agrobac- 
terium-mediated infiltrations, have been used to reconstitute a phytohormone, ABA signaling pathways, and quantitatively measure the function of each signaling component [8-10].

Transient gene expression in protoplasts is one of the most popular transient systems for the study of plant signaling pathways [11]. This system consists of a sensitive reporter specific for the signal of interest, in addition to a series of constructs to overexpress individual signaling components in protoplasts, which requires an efficient protoplast isolation and transfection method. Of all the phytohormone cascades, the abscisic acid (ABA) signaling pathway has been the most successfully reconstituted in Arabidopsis and rice protoplasts, allowing for a quantitative assessment of the effects of each signaling component $[8,9]$. However, other phytohormone signaling cascades have yet to be dissected to the same extent as that of ABA.

The phytohormone cytokinin (CK) modulates cell division, senescence, and chloroplast development $[12,13]$. The CK signaling pathway shares similarity with bacterial two-component signaling (TCS), which transduces various external environmental stimuli to affect gene expression $[14,15]$. CK signaling has evolved into a more complex system that includes additional signaling intermediates, leading to so-called multistep phosphorelay (MSP) regulation [16]. CK signaling comprises three critical signaling modules, the CK receptors, including histidine kinases (HKs), histidine phosphotransfer proteins (HPs), and type-B response regulators (type-B RRs) $[17,18]$. CK receptors are transmembrane proteins located at the plasma membrane or the endoplasmic reticulum membrane [19]. The binding of CK to the receptor activates the autophosphorylation activity of the HK at a His residue, which is followed by the transfer of the phosphoryl group to an Asp residue at the $\mathrm{C}$-terminus of the $\mathrm{CK}$ receptor. The phosphoryl group is then transmitted to a His residue on the HP, from which it is finally transferred to an Asp residue on type-B RRs in the nucleus $[20,21]$. Activated type-B RRs induce the transcription of CK-responsive genes by binding to the $\mathrm{CK}$ response motif (CRM, $\left.5^{\prime}-(\mathrm{A} / \mathrm{G}) \mathrm{GAT}(\mathrm{T} / \mathrm{C})-3^{\prime}\right)$ present in their promoters [22]. Müller and Sheen reported that a synthetic promoter consisting of multiple copies of the CRM motif driving the expression of the firefly luciferase (fLUC) gene acted as a sensitive CK reporter of type-B RR transcriptional activity [23]. This CK reporter was later further optimized, resulting in the new synthetic promoter TCSn (Two Component signaling Sensor new), which provided an attractive and easy readout of CK signaling in rice and maize (Zea mays) when driving the expression of the Green Fluorescent Protein (GFP) gene in the TCSn::GFP reporter [24]. The synthetic TCSn promoter was also used to drive the expression of $\beta$-GLUCURONIDASE (GUS) to reveal CK signaling in the root after treatment with 6-benzylaminopurine (BA) [25]. The TCSn promoter is therefore an efficient $\mathrm{CK}$ sensor that can be combined with different reporter genes to monitor CK signaling in diverse tissues. Recently, a new version of a CK-responsive promoter was also developed, referred to as TCSv2 [26].

In this study, we attempted to reconstitute the CK signaling cascade and identify the effects of each signaling component in rice protoplasts. We transiently cotransfected protoplasts with constructs overexpressing the three CK signaling components (HK, HP, and type-B RR) in addition to the CK sensor TCSn::LUC in various combinations. We monitored the activation of CK signaling, as determined by luciferase activity. Our results illustrate the applicability and usefulness of this system to discriminate the contribution of each CK signaling protein in relation to its signaling partners within networks and will be instrumental in deciphering the role of other components of the CK signaling cascade.

\section{Results}

\subsection{TCSn::fLUC Is a CK-Responsive Reporter in Rice Protoplasts}

The synthetic TCSn promoter consists of 16 copies of the ARR1A motif (5'-NGATT-3'), which is recognized by the type-B RR binding site, and induces the expression of reporter genes in a CK-dependent manner in both Arabidopsis and rice [25]. We cloned the TCSn promoter into a vector harboring the firefly luciferase gene ( $f L U C)$ to generate a versatile reporter for CK signaling in rice protoplasts. First, we tested whether this promoter 
induced luciferase activity specifically in response to CK treatment in rice protoplasts. To this end, we treated transiently transfected rice protoplasts expressing TCSn::fLUC and pUbi10::rLUC with major phytohormones individually at a final concentration of $5 \mu \mathrm{M}$. Only $5 \mu \mathrm{M}$ BA resulted in a fivefold increase in luciferase activity over mock controls. Other phytohormones failed to raise LUC activity significantly over mock controls, demonstrating the specificity of the TCSn::fLUC reporter for CK (Figure 1A). We also tested the TCSn::fLUC reporter with other active $\mathrm{CKs}$, kinetin (KT) and trans-zeatin (tZ), together with BA; all three CKs induced LUC activity to the same extent, although BA appeared to be slightly less potent at lower concentrations (Figure 1B). In addition, luciferase activity increased with higher $\mathrm{CK}$ concentrations of up to $20 \mu \mathrm{M}$ (Figure 1B).
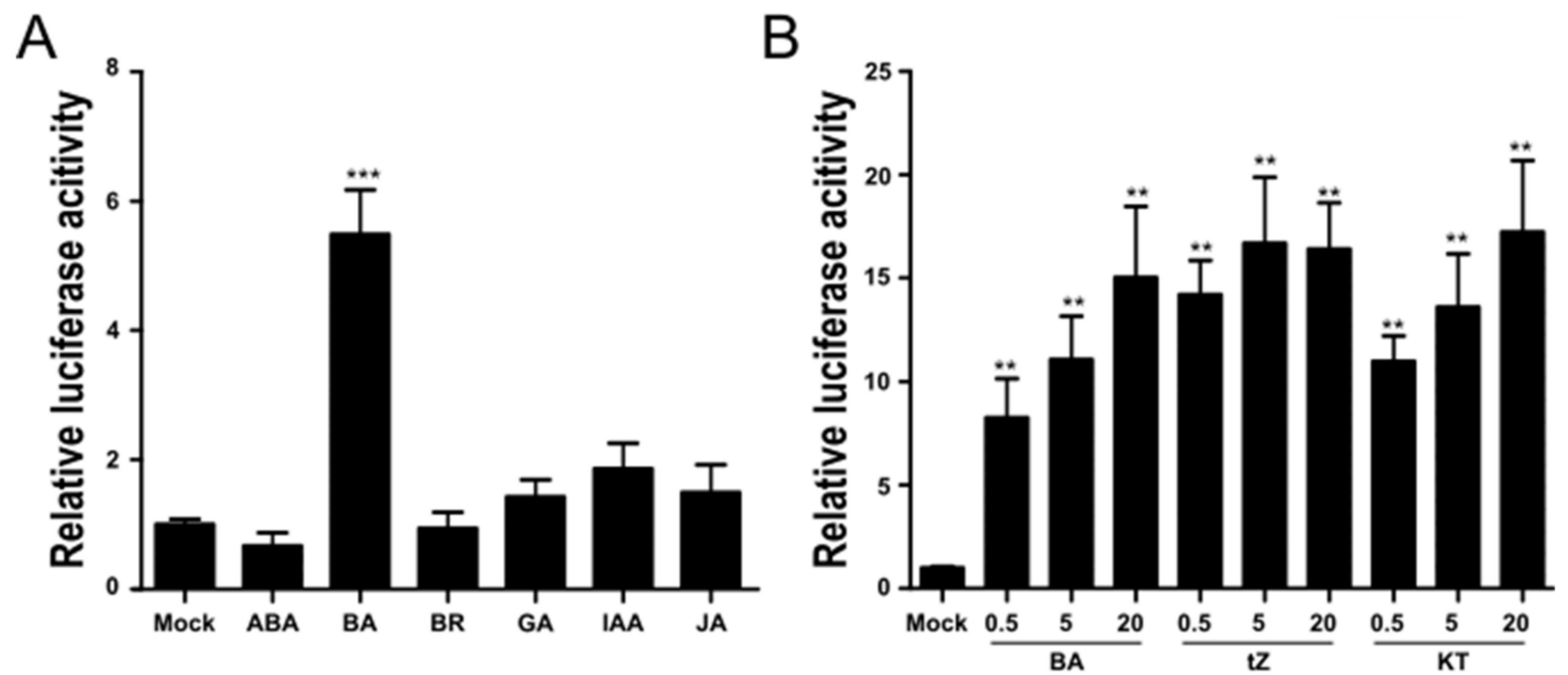

Figure 1. The TCSn::fLUC reporter specifically responds to CK in rice protoplasts. (A) TCSn::fLUC and OsUbi10::rLUC constructs were transiently transfected into rice protoplasts using the PEG transformation method. Transfected protoplasts were then exposed to $5 \mu \mathrm{M}$ ABA, IAA, BR, BA, GA, or JA. Firefly luciferase activity was measured $18 \mathrm{~h}$ after transfection. Statistical significance was determined by one-way ANOVA with Tukey's test, ${ }^{* * *} p<0.001$. (B) Rice protoplasts transiently transfected with TCSn::fLUC and OsUbi10::rLUC were treated with 0.5, 5, and $20 \mu \mathrm{M}$ of CKs BA, tZ, and KT. $N=6$. Statistical significance was determined by $t$-test, ${ }^{* *} p<0.01$. Luciferase activity was measured by comparing with a cotransformed marker, fLUC/rLUC. Values represent averages, and error bars are SEM of three independent biological repeats.

\subsection{Type-B OsRRs Have Different Trans Activities and CK Responsiveness for TCSn Promoter}

Type-B response regulators (RRs) are the MYB transcription factors that bind to the $T C S n$ promoter and transcriptionally induce their downstream genes [20]. The TCSn promoter, therefore, allowed us to monitor the transcriptional activity of each type-B OsRR in mock-treated or CK-treated protoplasts. Accordingly, we transfected rice protoplasts with TCSn:fLUC and effector constructs individually overexpressing the type-B OsRR genes OsRR16, OsRR17, OsRR18, and OsRR19. All tested genes strongly induced fLUC activity in an effector DNA dose-dependent manner (Figure 2A). Of all the genes tested here, overexpression of OsRR16 produced the highest fLUC activity irrespective of BA, and overexpression of OsRR17 showed the lowest response to BA. In contrast to OsRR16 and OsRR17, the overexpression of OsRR18 and OsRR19 resulted in the biggest fold induction of fLUC activity upon BA treatment relative to mock controls (Figure 2A). We determined the subcellular localization of OsRR-GFP fusion proteins to show whether proteins are translated well in protoplasts and have correct localization. All four proteins appeared to accumulate to similar levels, based on fluorescence intensity, and localized to the nucleus, as expected for transcription factors (Figure 2B). 

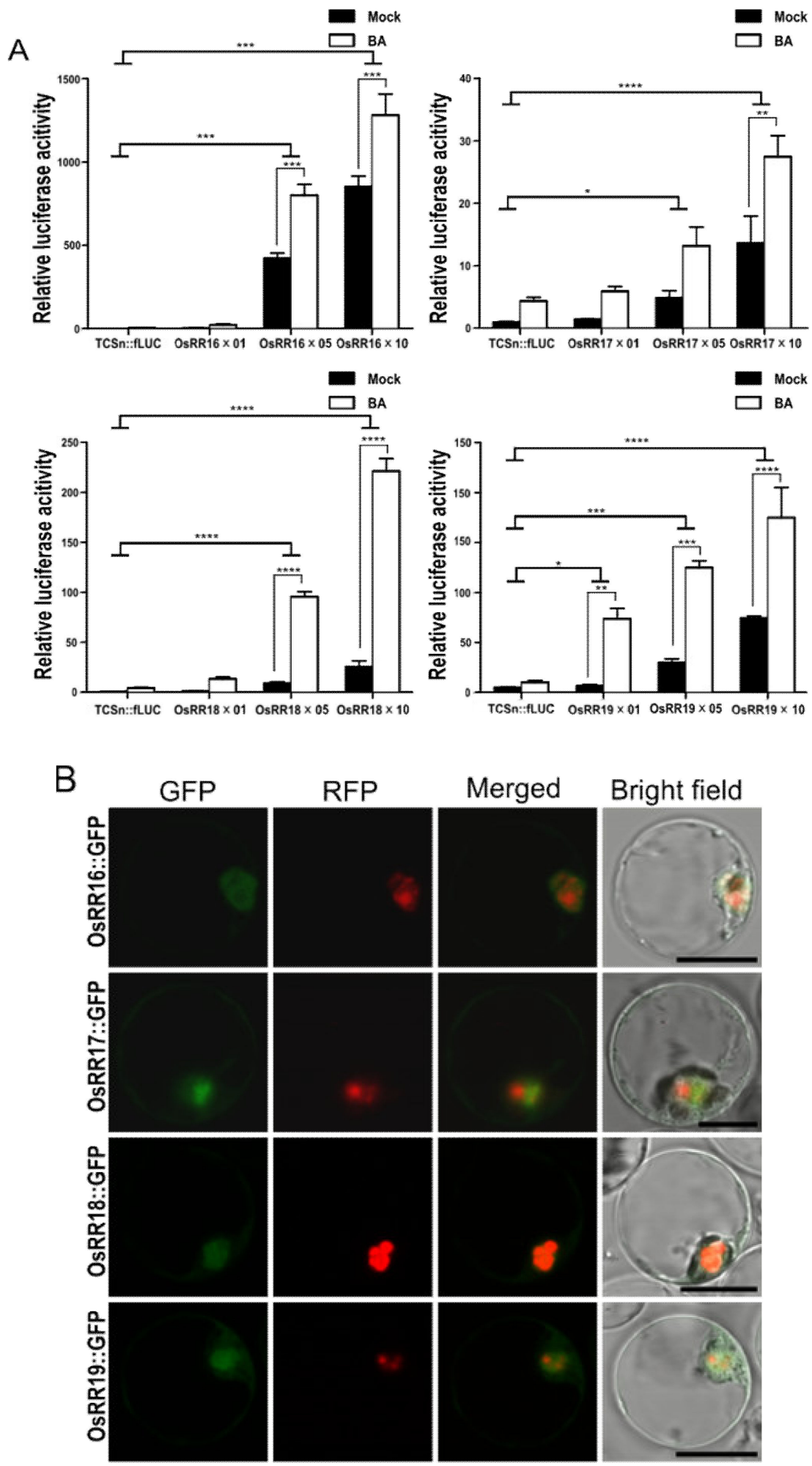

Figure 2. Different transcriptional activations of the TCSn promoter by type-B OsRRs in rice protoplasts. (A) All type-B OsRRs tested induce the transcription of fLUC from the TCSn promoter, as indicated by fLUC activity, in an effector DNA concentration-dependent manner. Labels along the $x$-axis $(\times 01, \times 05$, and $\times 10)$ indicate the amount of DNA used for transfection $(0.1,0.5$, and $1.0 \mu \mathrm{g}$, respectively). Transfected protoplasts were then exposed to $5 \mu \mathrm{M}$ BA. Firefly luciferase activity was measured $18 \mathrm{~h}$ after transfection. Luciferase activity was measured by comparing with a cotransformed marker, fLUC/rLUC. Values represent averages, and error bars are SEM of three independent biological repeats. Statistical significance was determined by two-way ANOVA with Tukey's multiple 
comparison test, ${ }^{*} p<0.05,{ }^{* *} p<0.01,{ }^{* * *} p<0.001,{ }^{* * * *} p<0.0001$. LUC activity measured from mocktreated samples was set to 1. (B) Subcellular localization of OsRR16-GFP, OsRR17-GFP, OsRR18-GFP, and OsRR19-GFP in rice protoplasts. NLS-RFP was used as a nuclear marker. Image was magnified by $400 \times$. Scale bar is $10 \mu \mathrm{m}$.

\subsection{Each HP Protein Increases the Transcriptional Activation of the TCSn Promoter by Type-B OsRRs}

Histidine phosphotransfer proteins (HPs) relay the phosphoryl group from the CK receptor to type-B OsRRs, leading to their activation. The overexpression of OsHP01 or OsHP02 alone in rice protoplasts weakly induced fLUC activity, depending on DNA concentration irrespective of CK. By contrast, overexpression of OsHP05 failed to significantly raise fLUC activity over levels measured for TCSn:fLUC alone up to $5 \mu \mathrm{M}$ DNA concentration (Figure 3A). To monitor the effects of OsHPs for type-B OsRRs, we next cotransfected each OsHP with a type-B OsRR as a pair. Notably, OsHP02 overexpression enhanced the transcriptional activity conferred by OsRR17 onto the TCSn::fLUC reporter. The transcriptional activation of the TCSn::fLUC reporter by OsRR18 increased significantly in response to BA treatment when coexpressed with OsHP01. However, overexpression of OsHP05 had no effect on the transcriptional activation of the TCSn::fLUC reporter by type-B OsRRs and appeared to significantly suppress the transcriptional activation of the reporter by OsRR17 with or without BA treatment (Figure 3B). Notably, OsHPs were not able to increase the reporter activity induced by OsRR19. We also noticed that all three OsHPs reduced the transcriptional activation of the reporter when they were coexpressed with OsRR17 in the absence of BA; as a result, the specificity of OsRR17 for BA increased. Because the OsHPs tested here differentially activated OsRRs in our assays and Dortay et al. 2006 reported that HPs are central hubs in interaction among CK signaling components in Arabidopsis using yeast two-hybrid assay, we assessed the interaction between OsRR17 and OsHPs by bimolecular fluorescence complementation (BiFC) [27]. All the three combinations tested (OsRR17-VN and OsHP01-VC or OsHP02-VC or OsHP05-VC) showed fluorescence from the Venus variant of GFP, confirming their physical association in planta in the nucleus (Supplementary Materials Figure S1).

\subsection{The CK Receptor OsHK03 Requires OsHPs to Induce the TCSn:fLUC Reporter}

The rice and Arabidopsis genomes both encode eight histidine kinases (HKs). However, three of them $(\mathrm{HK} 2,3,4)$ are actually CK receptors in Arabidopsis, and four of them are functional CK receptors in rice $[28,29]$. We cloned OsHKO3 and cotransfected the resulting construct with the TCSn::fLUC reporter into rice protoplasts. OsHK03 overexpression only weakly raised fLUC activity over that measured from the reporter alone in an effector DNA concentration- and BA-dependent manner. We then monitored the effect of cotransfecting OsHPs and $\mathrm{OsHKO3}$ in protoplasts on reporter activity. Basal transcriptional activation of the reporter significantly increased when OsHK03 was co-transfected with OsHP01 or OsHP02, but not with OsHP05, when compared with fLUC activity from OsHK03 transfected alone. Upon treatment with BA, the OsHK03/OsHP01 and OsHK03/OsHP02 pairs further increased reporter activity, in sharp contrast to the OsHK03/OsHP05 pair in which reporter activity showed no significant difference with or without BA (Figure 4). 

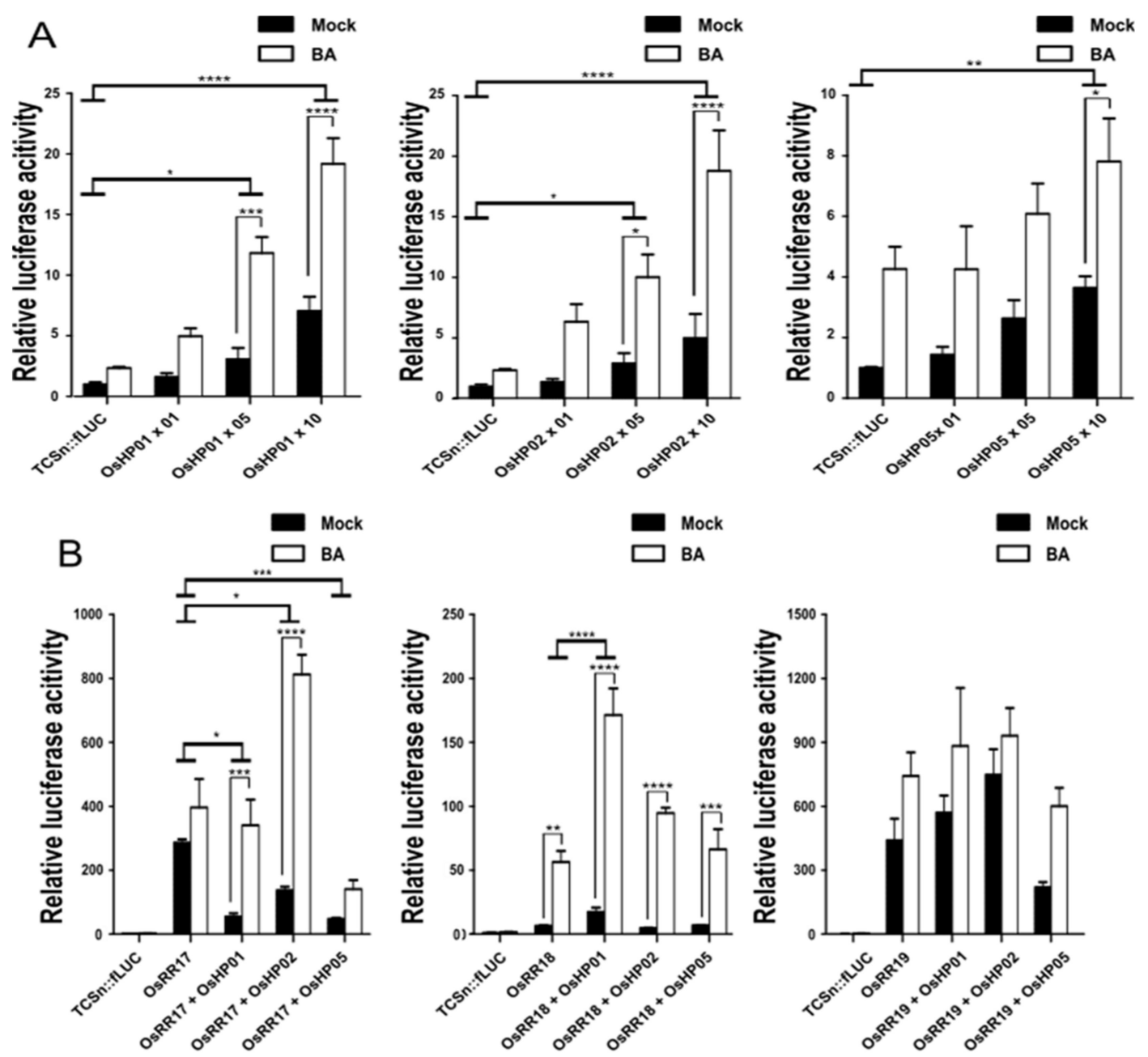

Figure 3. OsHPs modulate the transcriptional activity of type-B OsRRs in rice protoplasts. (A) The OsHP genes OsHP01, OsHP02, and OsHP05 were transiently overexpressed together with the TCSn::fLUC reporter. Labels along the $x$-axis $(\times 01, \times 05$, and $\times 10)$ indicate the amount of DNA used for transfection $(0.1,0.5$, and $1.0 \mu \mathrm{g}$, respectively). (B) Effect of cotransfection of OsHPs on the transcriptional activity of type-B OsRRs. OsRR17, OsRR18, or OsRR19 and OsHP1, OsHP2, or OsHP5 were cotransfected in pairs into rice protoplasts; fLUC activity was measured $18 \mathrm{~h}$ after transfection and $5 \mu \mathrm{M} B A$ treatment. Luciferase activity was measured by comparing with a cotransformed marker, fLUC/rLUC. Values represent averages, and error bars are SEM of three independent biological repeats. Statistical significance was determined by two-way ANOVA with Tukey's multiple comparison test, ${ }^{*} p<0.05,{ }^{* *} p<0.01,{ }^{* * *} p<0.001,{ }^{* * * *} P C<0.0001$. LUC activity measured from mock-treated samples was set to 1 . 

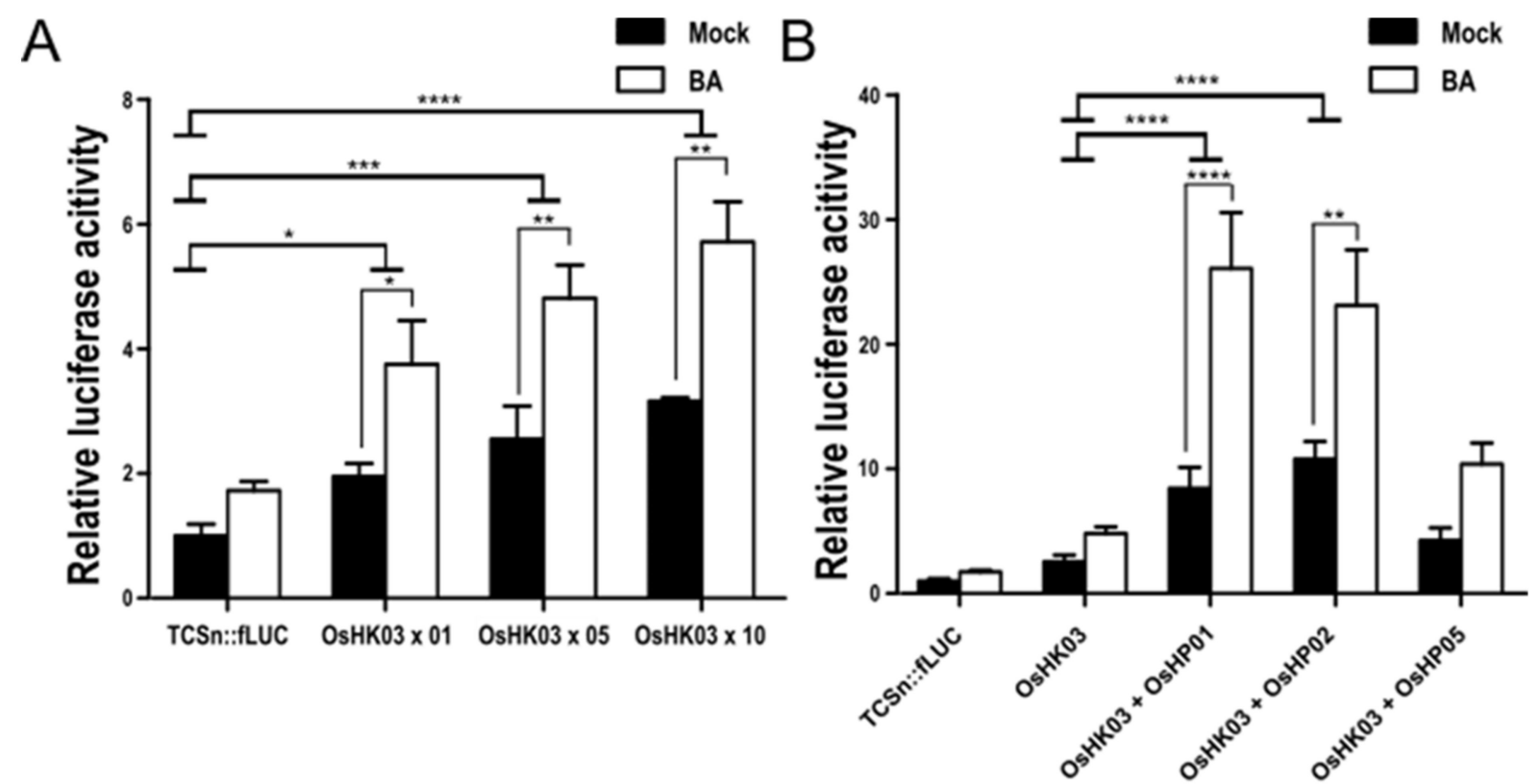

Figure 4. Cotransfection of OsHK03 and OsHPs increases transcriptional activity and sensitivity to BA. (A) Overexpression of OsHK03 weakly activates the TCSn::fLUC reporter. Labels along the $x$-axis (x 01, x 05, and $\mathrm{x} 10)$ indicate the amount of DNA used for transfection $(0.1,0.5$, and $1.0 \mu \mathrm{g}$, respectively). (B) Transcriptional activation of the reporter by overexpression of OsHK03 and three OsHPs, as measured by dual luciferase assay $18 \mathrm{~h}$ after transfection and $5 \mu \mathrm{M}$ BA treatment. Luciferase activity was measured by comparing with a cotransformed marker, fLUC/rLUC. Values represent averages, and error bars are SEM of three independent biological repeats. Statistical significance was determined by two-way ANOVA with Tukey's multiple comparison test, ${ }^{*} p<0.05,{ }^{* *} p<0.01,{ }^{* * *} p<0.001,{ }^{* * *} p<0.0001$. LUC activity measured from mock-treated samples was set to 1 .

\subsection{Reconstitution of the CK Signaling Pathway with Type-B OsRR, OsHP, and OsHK in Rice Protoplasts}

Finally, to reconstitute the entire CK transduction cascade in rice protoplasts with the described signaling intermediates cloned here, we transfected the three signaling components, type-B OsRRs, OsHPs, and OsHK alone, in pairs, or altogether (Figure 5). Cotransfection of OsRR17 with the reporter resulted in a strong induction of fLUC activity that was not BA dependent. The cotransfection of OsHK03 together with OsRR17 and the reporter construct did not increase fLUC activity further, suggesting that all three components are required to fully activate $\mathrm{CK}$ signaling. Indeed, the cotransfection of OsHK03, OsHP01, or OsHP02 and OsRR17 in rice protoplasts enhanced the basal transcriptional activation of the reporter about three- to fourfold over that seen with OsRR17 alone and conferred sensitivity to BA. As noted earlier with lower-order cotransfections, OsHP05 failed to activate the reporter when cotransfected with OsHK03 and OsRR17 and, in fact, lowered the activity of the reporter (Figure 5A). The overexpression of OsRR18 with OsHP01 and OsHK03 produced results comparable to those seen with OsRR17. Cotransfecting OsRR18 with OsHP05 and OsHK03 activated the reporter to the same level as OsRR18 alone, suggesting that OsRR18 does not participate in signaling with OsHK03 or OsHP05. Finally, cotransfecting OsRR19 together with OsHP02 and OsHK03 was the only combination of constructs that increased reporter activity and exhibited BA sensitivity, as neither OsHP01 nor OsHP05 activated the reporter (Figure 5C). 

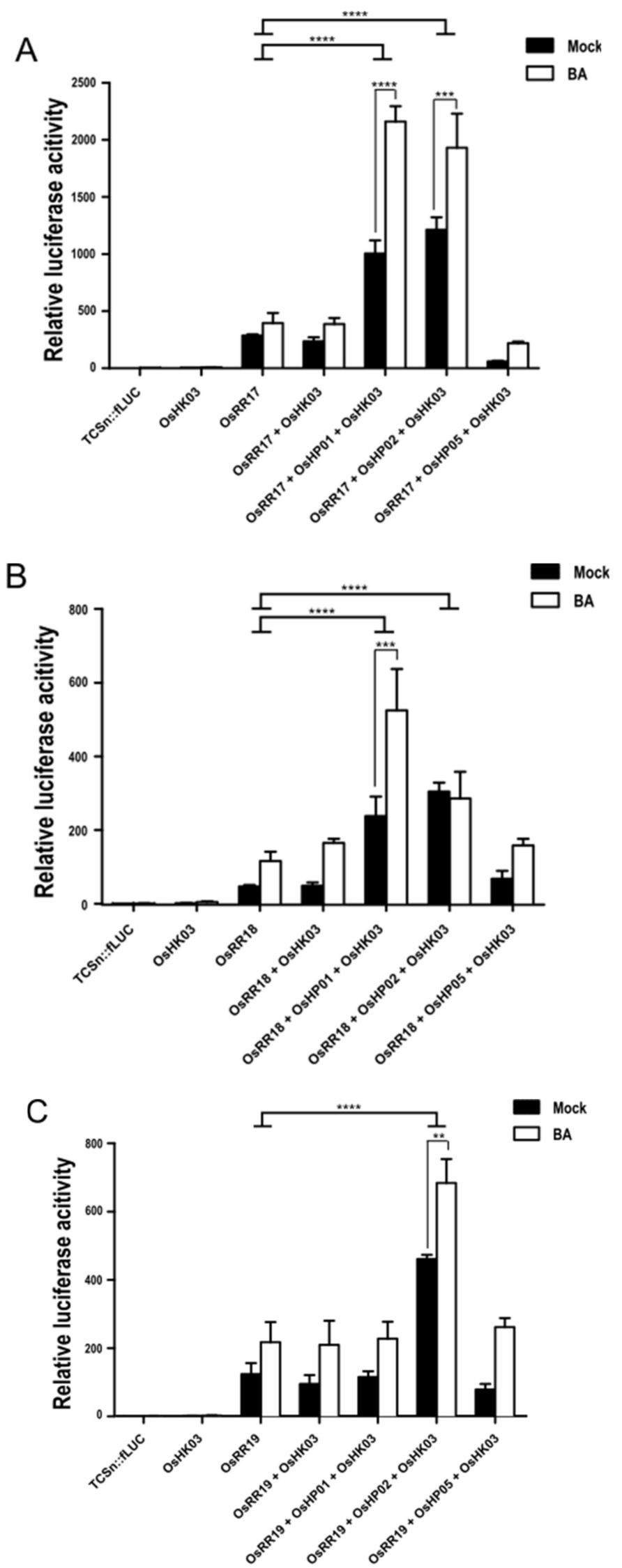

Figure 5. Reconstitution of CK signaling by overexpression of type-B OsRRs, OsHPs, and OsHK03 in rice protoplasts. Cotransfection of three transcription factor genes, OsRR17 (A), OsRR18 (B), and 
OsRR19 (C), with OsHPs and OsHK03 in rice protoplasts. The TCSn::fLUC and pUbi10::rLUC (a transformation control) reporter constructs were cotransfected with all other effectors. Transcriptional activation of the TCSn::fLUC reporter was determined $18 \mathrm{~h}$ after transfection and $5 \mu \mathrm{M}$ BA treatment. Luciferase activity was measured by comparing with a cotransformed marker, fLUC/rLUC. Values represent averages, and error bars are SEM of three independent biological repeats. Statistical significance was determined by two-way ANOVA with Tukey's multiple comparison test, and error bars indicate SEM, ${ }^{* *} p<0.01,{ }^{* * *} p<0.001,{ }^{* * * *} p<0.0001$. LUC activity measured from mock-treated samples was set to 1 .

\section{Discussion}

The major CK signaling components are evolutionarily conserved well across plant species, offering the possibility that the dissection of CK signaling in one plant species will be relevant to all plants. The rice genome encodes four HKs, five HPs (two functional and three pseudo HPs), and 16 type-B RRs. While Arabidopsis and rice have similar numbers of type-B RRs and HKs [18], rice is characterized by fewer functional HPs relative to Arabidopsis [30].

While many genetic studies have characterized the functions of CK-related genes in Arabidopsis and rice $[12,23,31]$, most phenotypes were reported from higher-order mutants to circumvent redundancy; it is therefore difficult to assign a specific function or activity to each gene in CK signaling [32]. The TCSn promoter was previously developed and applied to research on CK signaling in Arabidopsis, maize, and rice. In maize protoplasts, the TCSn promoter conferred a 15-fold induction of the reporter gene when treated with $\mathrm{tZ}$; in rice roots, GUS staining from TCSn::GUS reporter lines showed induction about sixfold when exposed to various CKs [23-25]. In our rice protoplast system, the TCSn::fLUC reporter vector alone displayed an induction around fivefold upon treatment with $\mathrm{CK}$, comparable to the TCSn::GUS results in rice roots. Coexpression of the type-B RR genes OsRR17, OsRR18, and OsRR19 induced the TCSn:fLUC reporter over 100-fold, thus providing a sensitive system in which to monitor the transcriptional activities of type-B RRs and reconstitute the $\mathrm{CK}$ signaling cascade. In this transient system, four type-B RRs exhibited distinct activities in terms of $\mathrm{CK}$ specificities and transcriptional activation of the reporter. Indeed, OsRR16 and OsRR17 did not activate the reporter in response to CK, whereas OsRR18 and OsRR19 strongly activated the reporter in response to CK. These results suggest that OsRR18 and OsRR19 might have specific functions at various developmental stages or affect tissues or phytohormone levels, which will await experimental validation.

The overexpression of OsHP or OsHK genes alone failed to significantly induce the activity of the TCSn::fLUC reporter. However, the cotransfection of all three components, OsHKs, OsHPs, and type-B OsRRs, significantly increased the reporter activity, supporting the notion that all three components are required for full activation of CK signaling. Endogenous CK signaling components are present in rice protoplasts and activate a CK-responsive reporter to interfere in the dissection of the activity of each signaling component. Heterologous systems, such as yeast or animal cell, can be used to avoid the background noises.

Of the three OsHPs tested here, OsHP05 had very low activity toward the reporter, or even suppressed CK responses. OsHP05 was reported to carry a Glu residue instead of His in the phosphotransfer domain, which might make OsHP5 a pseudo HP [33] (Supplementary Materials Figure S2). This hypothesis would be consistent with our results, as OsHP05 overexpression suppressed CK signaling, possibly via dominant-negative effects. Based on amino acid alignments, rice may therefore only have two functional HPs, OsHP1 and OsHP2, and both increased the transcriptional activity of the reporter significantly when cotransfected with the CK receptor [18]. Notably, OsHP01 and OsHP02 enhanced the transcriptional activation of the TCSn::fLUC reporter by OsRR17 in the absence of $\mathrm{CK}$ treatment. We interpret this observation as an indication that OsHP increases the CK-specific response of OsRR17. However, other type-B RRs did not show this behavior in relation to OsHPs, possibly because the reconstituted signaling cascade failed to deliver 
their cognate OsHPs; our conditions may also not have supplied each OsHP with its cognate type-B OsRR. Sun et al. 2014 showed that single knockdown lines for OsHP01 and OsHP02 exhibited significant developmental phenotypes during abiotic stress, supporting the notion that these HPs are functional [31]. However, functional differences between the two proteins were not explored, even though our results suggest that OsHP01 and OsHP02 might interact with different type-B OsRRs in terms of signaling network. The CK receptor did not induce CK signaling much when expressed alone or with type-B RRs in Arabidopsis or rice protoplasts [14]. We note here that cotransfection of type-B OsRR, OsHP, and OsHKs significantly increased the transcriptional activation of the TCSn::fLUC reporter already in the absence of exogenous CK treatment, while CK treatment only increased LUC activity two- to threefold further, suggesting that the endogenous CK content of protoplasts is sufficient to activate CK signaling in our conditions.

Based on the signaling activities observed here, we hypothesize that the OsHK03/OsHP01 and OsHP02/OsRR17 or OsHP02/OsRR18 pairs work alongside the same signaling network because their combination during transfection generated similar signaling activity. By contrast, OsRR19 does not appear to engage in a significant interaction with OsHP01, as its cotransfection with OsHPO1 and OsHKO3 weakly activated the reporter construct.

Plant signaling is very complex and relies on multilayered interactions. A better understanding can be obtained via bioinformatics analysis of gene families and gene expression profiles in response to genetic perturbations, from physical interaction networks, yeast two-hybrid assays, and pull-downs, as well as from proteomics and metabolomics, which require thorough, time-consuming integration. In this work, we aimed to reconstruct individual branches of $\mathrm{CK}$ signaling to dissect the effects of each component. The protoplast system allows high-throughput analysis in planta and shows low noise compared with whole plants. Luciferase activity can be easily monitored from transfected protoplasts and constitutes a very powerful tool to study CK signaling and inform candidate gene identification and analysis.

\section{Materials and Methods}

\subsection{Plant Materials and Growth Conditions}

The rice (Oryza sativa) cultivar Dongjin was used in this study. Hulled rice seeds were surface-sterilized with $70 \%$ ethanol for $30 \mathrm{~s}$, followed by $50 \% \mathrm{NaClO}$ containing a drop of Tween-20 for $15 \mathrm{~min}$ with constant agitation and five washes with distilled water. The $\mathrm{NaClO}$ sterilization step was repeated once but without Tween-20. The surface-sterilized seeds were thoroughly dried using sterilized dry filter paper. The seeds were then sown on half-strength Murashige and Skoog (1/2 MS) medium (supplemented with $0.4 \%$ Phytagel and adjusted to $\mathrm{pH}$ 5.8) and allowed to germinate for $10 \mathrm{~d}$ in the dark and $2 \mathrm{~d}$ in the light at $28^{\circ} \mathrm{C}$ before protoplast isolation.

\subsection{Isolation of Rice Protoplasts}

To isolate protoplasts from young rice seedling, shoots were sliced into $1 \mathrm{~mm}$ strips and incubated in enzyme solution (1.5\% Cellulase R-10 (Yakult Pharmaceutical Inc., Tokyo, JP), 0.75\% Macerozyme R-10 (Yakult Pharmaceutical Inc., Tokyo, JP), 0.6 M mannitol, and $10 \mathrm{mM}$ 2-(N-morpholino)ethanesulfonic acid (MES), pH 5.7 adjusting with $\mathrm{KOH}$ ) that had been activated at $65^{\circ} \mathrm{C}$ for $20 \mathrm{~min}$. The mixtures containing rice tissue and enzymes were incubated by shaking for $4 \mathrm{~h}$ at $28^{\circ} \mathrm{C}$, filtered on a $145 \mu \mathrm{m}$ mesh, and diluted with five volumes of W5 solution ( $154 \mathrm{mM} \mathrm{NaCl}, 125 \mathrm{mM} \mathrm{CaCl}_{2}, 5 \mathrm{mM} \mathrm{KCl}, 5 \mathrm{mM}$ glucose, and $3 \mathrm{mM}$ MES, pH 5.7 adjusting with $\mathrm{KOH}$ ). The diluted cell suspension was centrifuged at $100 \times g$ for $10 \mathrm{~min}$ at room temperature. The protoplast pellet was then resuspended in $3 \mathrm{~mL}$ of W5 solution and placed on top of $23 \%$ sucrose and centrifuged at $100 \times g$ for $10 \mathrm{~min}$ to remove remaining debris. The volume of separated protoplasts was then adjusted to $10 \mathrm{~mL}$ with W5 solution, and the cell density was determined on a hemocytometer. The protoplasts were centrifuged at $100 \times g$ for $7 \mathrm{~min}$ at room temperature and resuspended in 
MaMg solution (600 mM mannitol, $15 \mathrm{mM} \mathrm{MgCl}$, and $5 \mathrm{mM}$ MES, pH 5.7 adjusting with $\mathrm{KOH}$ ) for transfection.

\subsection{Transient Gene Expression in Rice Protoplast}

Protoplasts $\left(4-5 \times 10^{4}\right.$ cells) were suspended in $300 \mu \mathrm{L}$ MaMg solution and mixed with up to $30 \mu \mathrm{g}$ of DNA and $40 \%$ PEG solution ( $400 \mathrm{mM}$ mannitol, $100 \mathrm{mM} \mathrm{Ca}\left(\mathrm{NO}_{3}\right)_{2}$, and $40 \%$ PEG 6000). The solution was mixed thoroughly using a fixed-angle rotating wheel for $10 \mathrm{~min}$ and incubated for $30 \mathrm{~min}$ at room temperature. After incubation, $630 \mu \mathrm{L}$, $1.25 \mathrm{~mL}$, and $2.5 \mathrm{~mL}$ of W5 solution were added every $10 \mathrm{~min}$ over the course of $30 \mathrm{~min}$. The mixtures were incubated for another $25 \mathrm{~min}$ and centrifuged at $100 \times g$ for $5 \mathrm{~min}$ to remove the PEG solution. The protoplast pellets were resuspended in $3 \mathrm{~mL}$ of W5 solution and incubated at $28^{\circ} \mathrm{C}$ overnight $(18 \mathrm{~h})$ with or without CKs in the dark.

\subsection{Construction of Vectors}

We selected the synthetic Two Component signaling Sensor new (TCSn) promoter as a reporter in rice, as it specifically responds to CK [24]. The TCSn promoter was cloned upstream of the firefly luciferase $(f L U C)$ gene to generate a CK reporter. The coding sequences for various $C K$ signaling components (type-B response regulator (RR), histidine phosphotransfer protein (HP), and histidine kinase (HK)) were PCR-amplified, cloned into the pENTRY D-Topo vector (Invitrogen, Carlsbad, CA, USA), and then recombined into destination vectors for signaling effectors, subcellular localization, and bimolecular fluorescence complementation (BiFC) assays by LR recombination (Invitrogen, Carlsbad, CA, USA) as previously described [8]. The primers used are listed in Supplementary Materials Table S1.

\subsection{Dual Luciferase Assays}

For dual luciferase assays, TCSn:fLUC, Renilla luciferase driven by the ubiquitin promoter ( $p$ Ubi10::rLUC), and effector constructs were introduced into rice protoplasts by PEG-mediated transfection. After $18 \mathrm{~h}$ of incubation, the protoplasts were centrifuged at $300 \mathrm{~g}$ for $30 \mathrm{~s}$ and the supernatants removed. Pelleted cells were resuspended in $50 \mu \mathrm{L}$ $1 \times$ passive lysis buffer (PLB). Renilla and firefly luciferase activities were measured with $10 \mu \mathrm{L}$ lysate according to the manufacturer's protocol (Promega, Madison, WI, USA); firefly luciferase activity was then normalized to Renilla luciferase activity to account for transfection efficiency.

\subsection{Subcellular Localization and Bimolecular Fluorescence Complementation (BiFC) Assay}

To determine the localization of type-B OsRRs in plant cells, we transfected rice protoplasts with OsRR-GFP fusion constructs. Red fluorescent protein (RFP) with a nuclear localization sequence (NLS) was used as nucleus-localized control. GFP fluorescence (493-546 nm) was observed and imaged using a Leica TCS SP8 confocal microscope (Leica Microsystems, Germany). For BiFC assays, OsRR17 was fused to the N-terminus of the Venus variant of GFP (OsRR17-VN), while three OsHPs were fused to the C-terminus of Venus (OsHP-VC). The corresponding constructs were transfected in rice protoplasts as above. OsbZIP10-VC was used as a negative control, and NLS-RFP was used as a nucleuslocalized control. RFP fluorescence (581-652 nm) was observed and imaged using a Leica TCS SP8 confocal microscope.

Supplementary Materials: The following are available online at https:/ /www.mdpi.com/article/10 $.3390 /$ ijms22073647/s1.

Author Contributions: E.G. and B.-G.K. designed the research plants; J.-Y.L., S.B.L., and B.-G.K. supervised the experiments; J.S. cultivated the rice; J.H., M.K.M., S.P. and E.G. performed the rice protoplast isolation; E.G. cloned the CK signaling genes and gene expression analysis; E.G. and B.-G.K. wrote the manuscript; J.-Y.L. and S.B.L. edited the manuscript. All authors have read and agreed to the published version of the manuscript. 
Funding: This work was supported by the Agenda Program (PJ014838) and New Breeding Technologies Development Program (PJ014773) of the Rural Development Administration.

Conflicts of Interest: The authors declare no conflict of interest.

\section{References}

1. Aoyama, T.; Oka, A. Cytokinin signal transduction in plant cells. J. Plant Res. 2003, 116, 221-231. [CrossRef] [PubMed]

2. Blazquez, M.A.; Nelson, D.C.; Weijers, D. Evolution of Plant Hormone Response Pathways. Annu. Rev. Plant Biol. 2020, 71, 327-353. [CrossRef] [PubMed]

3. McSteen, P.; Zhao, Y. Plant hormones and signaling: Common themes and new developments. Dev. Cell 2008, 14, 467-473. [CrossRef] [PubMed]

4. Wang, C.; Liu, Y.; Li, S.S.; Han, G.Z. Insights into the origin and evolution of the plant hormone signaling machinery. Plant Physiol. 2015, 167, 872-886. [CrossRef] [PubMed]

5. Miao, C.; Xiao, L.; Hua, K.; Zou, C.; Zhao, Y.; Bressan, R.A.; Zhu, J.K. Mutations in a subfamily of abscisic acid receptor genes promote rice growth and productivity. Proc. Natl. Acad. Sci. USA 2018, 115, 6058-6063. [CrossRef]

6. Zhao, Y.; Zhang, Z.; Gao, J.; Wang, P.; Hu, T.; Wang, Z.; Hou, Y.J.; Wan, Y.; Liu, W.; Xie, S.; et al. Arabidopsis Duodecuple Mutant of PYL ABA Receptors Reveals PYL Repression of ABA-Independent SnRK2 Activity. Cell Rep. 2018, 23, 3340-3351.e3345. [CrossRef]

7. Kim, H.; Hwang, H.; Hong, J.W.; Lee, Y.N.; Ahn, I.P.; Yoon, I.S.; Yoo, S.D.; Lee, S.; Lee, S.C.; Kim, B.G. A rice orthologue of the ABA receptor, OsPYL/RCAR5, is a positive regulator of the ABA signal transduction pathway in seed germination and early seedling growth. J. Exp. Bot. 2012, 63, 1013-1024. [CrossRef]

8. Kim, N.; Moon, S.J.; Min, M.K.; Choi, E.H.; Kim, J.A.; Koh, E.Y.; Yoon, I.; Byun, M.O.; Yoo, S.D.; Kim, B.G. Functional characterization and reconstitution of ABA signaling components using transient gene expression in rice protoplasts. Front. Plant Sci. 2015, 6, 614. [CrossRef]

9. Fujii, H.; Chinnusamy, V.; Rodrigues, A.; Rubio, S.; Antoni, R.; Park, S.Y.; Cutler, S.R.; Sheen, J.; Rodriguez, P.L.; Zhu, J.K. In vitro reconstitution of an abscisic acid signalling pathway. Nature 2009, 462, 660-664. [CrossRef] [PubMed]

10. Ruschhaupt, M.; Mergner, J.; Mucha, S.; Papacek, M.; Doch, I.; Tischer, S.V.; Hemmler, D.; Chiasson, D.; Edel, K.H.; Kudla, J.; et al. Rebuilding core abscisic acid signaling pathways of Arabidopsis in yeast. EMBO J. 2019, 38, e101859. [CrossRef] [PubMed]

11. Yoo, S.D.; Cho, Y.H.; Sheen, J. Arabidopsis mesophyll protoplasts: A versatile cell system for transient gene expression analysis. Nat. Protoc. 2007, 2, 1565-1572. [CrossRef] [PubMed]

12. Müller, B. Generic signal-specific responses: Cytokinin and context-dependent cellular responses. J. Exp. Bot. 2011, 62, 3273-3288. [CrossRef] [PubMed]

13. Sheen, J. Phosphorelay and transcription control in cytokinin signal transduction. Science 2002, 296, 1650-1652. [CrossRef] [PubMed]

14. Hwang, I.; Sheen, J. Two-component circuitry in Arabidopsis cytokinin signal transduction. Nature 2001, 413, 383-389. [CrossRef] [PubMed]

15. To, J.P.; Kieber, J.J. Cytokinin signaling: Two-components and more. Trends Plant Sci. 2008, 13, 85-92. [CrossRef] [PubMed]

16. Mira-Rodado, V. New Insights into Multistep-Phosphorelay (MSP)/ Two-Component System (TCS) Regulation: Are Plants and Bacteria that Different? Plants 2019, 8, 590. [CrossRef]

17. Du, L.; Jiao, F.; Chu, J.; Jin, G.; Chen, M.; Wu, P. The two-component signal system in rice (Oryza sativa L.): A genome-wide study of cytokinin signal perception and transduction. Genomics 2007, 89, 697-707. [CrossRef]

18. Tsai, Y.C.; Weir, N.R.; Hill, K.; Zhang, W.; Kim, H.J.; Shiu, S.H.; Schaller, G.E.; Kieber, J.J. Characterization of genes involved in cytokinin signaling and metabolism from rice. Plant Physiol. 2012, 158, 1666-1684. [CrossRef]

19. Romanov, G.A.; Lomin, S.N.; Schmulling, T. Cytokinin signaling: From the ER or from the PM? That is the question! New Phytol. 2018, 218, 41-53. [CrossRef]

20. Hwang, I.; Sheen, J.; Müller, B. Cytokinin signaling networks. Annu. Rev. Plant Biol. 2012, 63, 353-380. [CrossRef]

21. Schaller, G.E.; Kieber, J.J.; Shiu, S.H. Two-component signaling elements and histidyl-aspartyl phosphorelays. Arab. Book 2008, 6, e0112. [CrossRef] [PubMed]

22. Ramireddy, E.; Brenner, W.G.; Pfeifer, A.; Heyl, A.; Schmulling, T. In planta analysis of a cis-regulatory cytokinin response motif in Arabidopsis and identification of a novel enhancer sequence. Plant Cell Physiol. 2013, 54, 1079-1092. [CrossRef] [PubMed]

23. Müller, B.; Sheen, J. Cytokinin and auxin interaction in root stem-cell specification during early embryogenesis. Nature 2008, 453 , 1094-1097. [CrossRef] [PubMed]

24. Zürcher, E.; Tavor-Deslex, D.; Lituiev, D.; Enkerli, K.; Tarr, P.T.; Müller, B. A robust and sensitive synthetic sensor to monitor the transcriptional output of the cytokinin signaling network in planta. Plant Physiol. 2013, 161, 1066-1075. [CrossRef] [PubMed]

25. Tao, J.; Sun, H.; Gu, P.; Liang, Z.; Chen, X.; Lou, J.; Xu, G.; Zhang, Y. A sensitive synthetic reporter for visualizing cytokinin signaling output in rice. Plant Methods 2017, 13, 89. [CrossRef]

26. Steiner, E.; Israeli, A.; Gupta, R.; Schwartz, I.; Nir, I.; Leibman-Markus, M.; Tal, L.; Farber, M.; Amsalem, Z.; Ori Naomi Müller, B.; et al. Characterization of the cytokinin sensor TCSv2 in arabidopsis and tomato. Plant Methods 2020, 16, 1-12. [CrossRef]

27. Dortray, H.; Mehnert, N.; Burkle, L.; Schmulling, T.; Hey, A. Analysis of protein interactions within the cytokinin-signaling pathway of Arabidopsis thaliana. FEBS J. 2006, 273, 4631-4644. [CrossRef] 
28. Nishiyama, R.; Watanabe, Y.; Leyva-Gonzalez, M.A.; Ha, C.V.; Fujita, Y.; Tanaka, M.; Seki, M.; Yamaguchi-Shinozaki, K.; Shinozaki, K.; Herrera-Estrella, L.; et al. Arabidopsis AHP2, AHP3, and AHP5 histidine phosphotransfer proteins function as redundant negative regulators of drought stress response. Proc. Natl. Acad. Sci. USA 2013, 110, 4840-4845. [CrossRef] [PubMed]

29. Schaller, G.E.; Shiu, S.; Armitage, P.A. Two-Component Systems and Their Co-Option for Eukaryotic Signal Transduction. Curr. Biol. 2011, 21, R320-R330. [CrossRef]

30. Hutchison, C.E.; Li, J.; Argueso, C.; Gonzalez, M.; Lee, E.; Lewis, M.W.; Maxwell, B.B.; Perdue, T.D.; Schaller, G.E.; Alonso, J.M.; et al. The Arabidopsis histidine phosphotransfer proteins are redundant positive regulators of cytokinin signaling. Plant Cell 2006, 18, 3073-3087. [CrossRef]

31. Sun, L.; Zhang, Q.; Wu, J.; Zhang, L.; Jiao, X.; Zhang, S.; Zhang, Z.; Sun, D.; Lu, T.; Sun, Y. Two rice authentic histidine phosphotransfer proteins, OsAHP1 and OsAHP2, mediate cytokinin signaling and stress responses in rice. Plant Physiol. 2014, 165, 335-345. [CrossRef] [PubMed]

32. Jeon, J.; Kim, J. Arabidopsis response Regulator1 and Arabidopsis histidine phosphotransfer Protein2 (AHP2), AHP3, and AHP5 function in cold signaling. Plant Physiol. 2013, 161, 408-424. [CrossRef] [PubMed]

33. Mähönen, A.P.; Bishopp, A.; Higuchi, M.; Nieminen, K.M.; Kinoshita, K.; Tormakangas, K.; Ikeda, Y.; Oka, A.; Kakimoto, T.; Helariutta, Y. Cytokinin signaling and its inhibitor AHP6 regulate cell fate during vascular development. Science 2006, 311, 94-98. [CrossRef] [PubMed] 